\title{
Validation of Hospital Performance Measures of Acute Stroke Care Quality. Riksstroke, the Swedish Stroke Register
}

\author{
Anna Söderholm Birgitta Stegmayr Eva-Lotta Glader Kjell Asplund for the \\ Riksstroke Collaboration
}

Riksstroke, Medicine, Department of Public Health and Clinical Medicine, Umeå University, Umeå, Sweden

\section{Key Words}

Stroke register - Validation - Data quality · Performance measurement data quality to permit between-hospital performance benchmarking. Our experiences may serve as a model for other stroke registers while evaluating data quality.

(c) 2016 S. Karger AG, Basel

\section{Introduction}

Registers are increasingly used to monitor and benchmark between-hospital or between-country stroke care performance [1-9]. However, the quality of data included in the registers is often insufficiently assessed. So, a fundamental question is: Is the quality of the register data good enough to permit fair assessments of stroke care quality and benchmarking? This question comes more in focus as the registers are increasingly used for reimbursements and research.

Riksstroke, the Swedish Stroke Register, is a national stroke care quality register established in 1994. Riksstroke records information in the acute phase of stroke and, by questionnaires to survivors at follow-ups during the 3rd and 12th month after stroke. In this article, we report validation studies on items recorded during in-hospital care for acute stroke. We have addressed 3 key validity issues: (i) completeness of recording in-hospital stroke events

\section{KARGER}

E-Mail karger@karger.com

www.karger.com/ned
(C) 2016 S. Karger AG, Basel

0251-5350/16/0464-0229\$39.50/0
Professor Kjell Asplund

Riksstroke, Medicine, Department of Public Health and Clinical Medicine Umeå University Hospital

SE-90185 Umeå (Sweden)

E-Mail kjellasplund1@gmail.se 


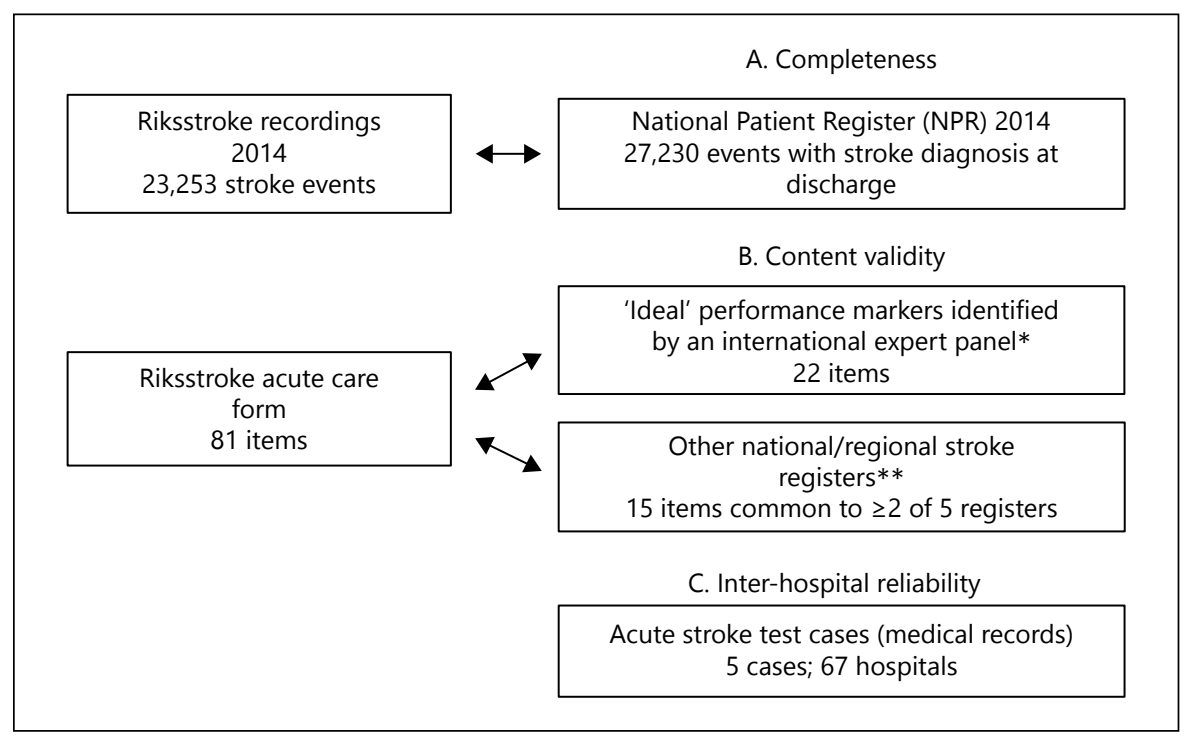

(so that the register gives an all-inclusive picture of stroke care quality), (ii) content validity (the extent to which the items recorded in Riksstroke is considered by stroke experts to be useful to measure the quality of care), and (iii) inter-hospital reliability (variations between different hospitals recording the same items).

\section{Methods}

The main features of Riksstroke have previously been published [6] and are presented in detail at the Riksstroke website www.riksstroke.org, as are registration forms.

Riksstroke was established in 1994 with the primary aim of serving as a national register monitoring the quality of stroke care and to stimulate improvement by providing health care providers with the 'Study' component of the Plan-Do-Study-Act cycle for quality improvement [10]. Riksstroke provides online feedback to participating hospitals with several benchmarking options and produces annual reports with comprehensive assessments of stroke care quality. At the national level, Riksstroke is an instrument to follow to what extent stroke guidelines issued by the National Board of Health and Welfare (the national governmental agency for health care) are adhered to [11]. A secondary aim of Riksstroke is to provide a database for research on stroke management.

The present report summarizes observations from studies evaluating items recorded in the acute phase of stroke. The evaluations were performed in 2012-2013 except evaluation of completeness, for which data from year 2014 was used. The validation studies are summarized in figure 1 .

To estimate completeness, events recorded in Riksstroke were individually matched with acute stroke diagnoses (ICD-10 codes I61, I63 and I64) at discharge from hospital in the National Patient Register (NPR) at the National Board of Health and Welfare. NPR is compulsory and comprises all admissions to hospital [12]. In the calculations of completeness, the number of events in Riksstroke was the nominator and the number of stroke events in NPR plus cases identified by Riksstroke but not by NPR was the denominator. Emergency room visits without admission were not included. Multiple hospitalization events with an acute stroke diagnosis occurring within 28 days of the index event were excluded. As previously reported, the accuracy of acute stroke diagnoses in NPR was validated by comparison with a population-based stroke research register (Northern Sweden MONICA Study) with individual assessment of each case [13].

Content validity was assessed by 2 sets of comparisons. First, we compared items recorded in Riksstroke with a core set of 22 stroke care performance measures identified by an international panel of stroke experts (stroke physicians, neurologists, public health academics and representatives from patient organisations) gathered under the auspices of European Implementation Score (EIS) project. This set of measures is intended to be used for crossnational comparisons of the quality of acute stroke care [14]. Second, we compared the Riksstroke variables with items that are recorded by other European stroke audits and registers (Belgium, England/Wales/Northern Ireland, Germany, Scotland, Spain), also within the EIS project; only the 15 variables that were used by at least 2 of the other 5 registers were included in this comparison [15].

Inter-hospital reliability was assessed by distributing a set of 5 anonymized test cases, taken from routine medical records in an abbreviated form (deleting redundant information but otherwise verbatim), to hospitals participating in Riksstroke, asking them to record the cases by their regular routines for acute stroke care (81 items). The purposes were (a) to identify variables with low interhospital reliability, and (b) to identify hospitals with a high proportion of deviant responses. Pre hoc, a working group of experienced stroke staff members agreed on what coding should be regarded as correct. For each item, overall reliability was thus calculated based on 5 cases in 67 hospitals ( 325 observations). Between-hospital variations were also calculated. 
Fig. 2. Between-hospital variation of completeness of all acute stroke cases by Riksstroke, calculated as described in Methods. Each horizontal column represents one hospital. Calculated without adjustment for possible false positive cases in the NPR.

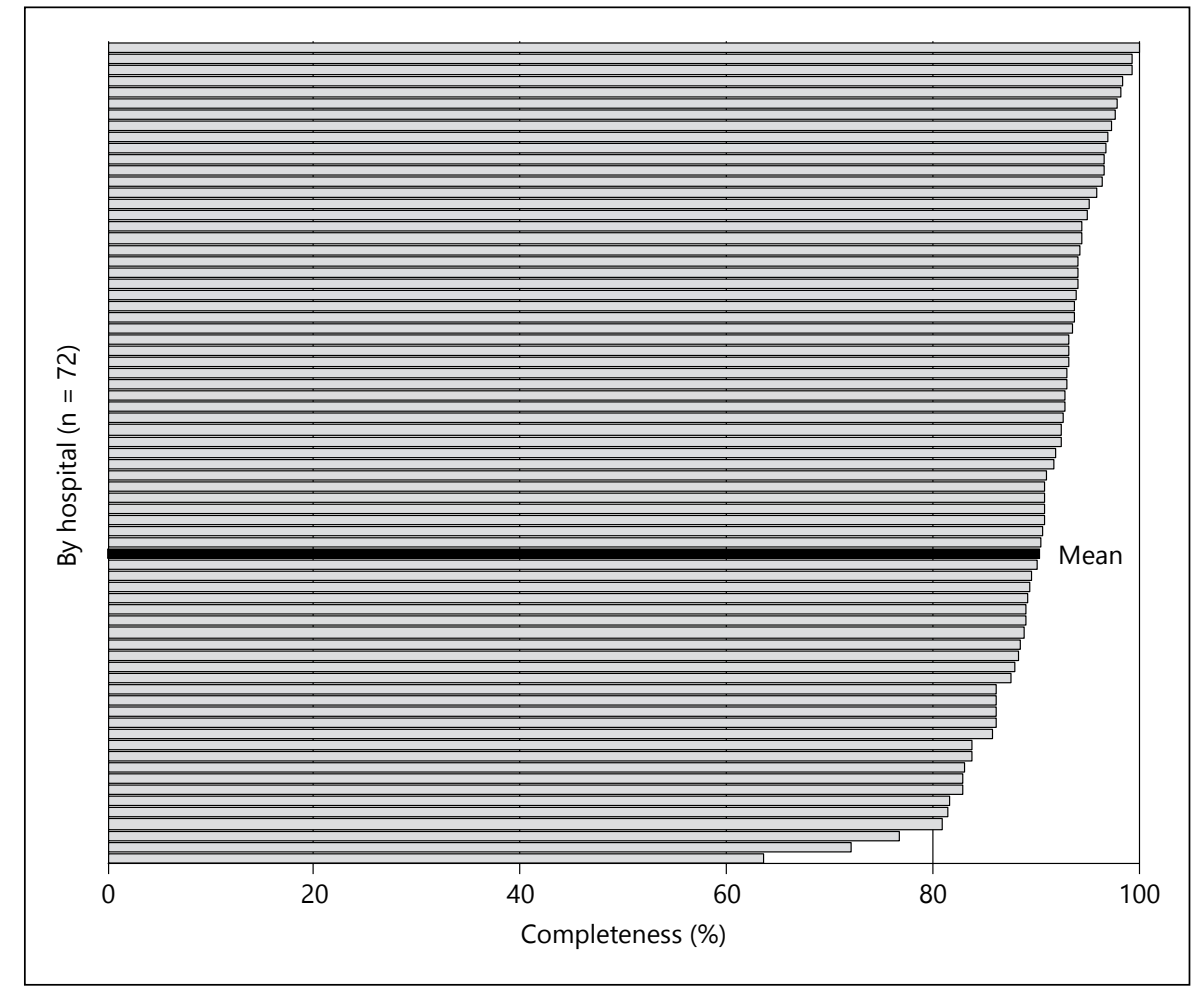

\section{Results}

\section{Completeness}

Since 1998, Riksstroke covers all hospitals in the country admitting acute stroke patients (72 hospitals in 2014). In 2014 , there was a total of 27,230 events with a diagnosis of acute stroke at hospital discharge in the national administrative register NPR or identified by Riksstroke but not NPR. Of these events, $23.253(89.5 \%)$ were recorded in Riksstroke. For first-ever stroke events, 18,440 of 20,421 (90.3\%) were recorded in Riksstroke. When the NPR was validated, the proportion of hospital admissions with a false positive diagnosis of acute stroke was $6 \%$ for first-ever events and $12 \%$ for all-stroke events [13]. The completeness by hospital is shown in figure 2 . Riksstroke has set 2 target levels for coverage (without adjustment for possible false positive cases in the NPR), moderate at $\geq 85 \%$ and high at $\geq 92 \%$. The high target level was reached by 37 of the 72 hospitals (51\%), whereas 24 hospitals (33\%) were in the $85-91 \%$ range and 11 hospitals (15\%) had coverage below $85 \%$.

\section{Content Validity}

To assess content validity in an international perspective, we compared items recorded in Riksstroke with the core set of quality indicators identified by an internation- al panel of stroke experts, as described in Methods. The panel defined a set of 22 quality indicators in 6 domains that ideally would be included in a stroke quality register to permit cross-country comparisons. In addition, 6 background variables to be used in case-mix adjustments were identified [14]. Table 1 shows to what extent Riksstroke covers this ideal set of indicators. Of the 22 variables identified by the international panel, 18 are included in Riksstroke. One item (dead or ADL dependent) is assessed at a different time point in Riksstroke (at 3 months) than proposed by the panel (30 days). Carotid surgery is recorded in the national vascular surgery register Swedevasc but not in Riksstroke. Early administration of aspirin (2 items proposed by the panel) is not recorded in Riksstroke.

In the next step to assess the content validity, we compared the Riksstroke variables with those in 5 other European stroke audits and registers included in EIS. The indicators used by the EIS registers were highly heterogeneous. There were 15 variables that were used in at least 2 of the registers. Riksstroke included 14 of them (table 1). The item not recorded in Riksstroke was early administration of aspirin. While some other European registers include assessment for depression during hospital stay, Riksstroke measures the self-assessed mood 3 months after stroke. 
Table 1. Quality indicators identified by international stroke experts within the framework of the EIS [14] and variables used by at least 2 of 5 other European stroke registers [8]. The right column shows to what extent these variables are covered by Riksstroke. Categorisation of variable domains as by EIS [14]

Key variables identified by international stroke experts
Variables in $\geq 2$ European Riksstroke stroke registers

\section{Coordination of care}

Stroke unit care

Length of stroke unit stay

Diagnostic procedures

Brain imaging

Time to first brain imaging

Carotid artery imaging

ECG recording

Assessment by physiotherapist/occupational therapist

Assessment of depression

Preservation of neuronal tissue

Early administration of aspirin

Thrombolysis

Door-to-needle time in thrombolysis

Prevention of complications

Test of swallowing

Initiation of secondary prevention

Antiplatelet drugs within $48 \mathrm{~h}$

Antiplatelet drugs at discharge

Oral anticoagulants at discharge after embolic stroke

Statins at discharge after ischemic stroke

Antihypertensive agents at discharge

Information on smoking cessation

Time to imaging of carotid arteries

Carotid surgery performed

Survival and functional outcome

Symptomatic brain hemorrhage after thrombolysis

Dead during hospital stay

Deceased within 30 days

Dead or ADL dependent at 30 days

$\mathrm{X}$
$\mathrm{X}$
$\mathrm{X}$
$\mathrm{X}$
$\mathrm{X}$
$\mathrm{X}$
$\mathrm{X}$

$\mathrm{X}$

$\mathrm{X}$

$\mathrm{X}$

$\mathrm{X}$

$\mathrm{X}$

$\mathrm{X}$

$\mathrm{X}$

$\mathrm{X}$

$\mathrm{X}$

$\mathrm{X}$

$\mathrm{X}$

$\mathrm{X}$

$\mathrm{X}$

$\mathrm{X}$

$\mathrm{X}$

Other

Early mobilisation

Background information

Age

Sex

Type of stroke

Destination at discharge

Level of consciousness

NIHSS at hospital admission
$\mathrm{X}$

$\mathrm{X}$

$\mathrm{X}$

$\mathrm{X}$

$\mathrm{X}$

$\mathrm{X}^{1}$

$\mathrm{X}$

$\mathrm{X}^{2}$

$\mathrm{X}$

$\mathrm{X}$

$\mathrm{X}$

X $\quad$ X

X $\quad X$

$\mathrm{X} \quad \mathrm{X}$

X $\quad$ X

$\mathrm{X}$

$\mathrm{X}$

$\mathrm{X}$

$\mathrm{X}$

$\mathrm{X}^{4}$

$\mathrm{X}$

X

$\mathrm{X}$

$\mathrm{X}$

$\mathrm{X}$

$\mathrm{X}$

$\mathrm{X}$

$\mathrm{X}$

${ }^{1}$ Long-term ECG monitoring recorded in Riksstroke, any ECG recorded in other registers and proposed by experts.

${ }^{2}$ Self-reported mood assessed at 3 months in Riksstroke, depression assessed during hospital stay in other registers.

${ }^{3}$ Detailed recording in the national vascular surgery register Swedevasc.

${ }^{4}$ Reported at 3 months in Riksstroke.

\section{Inter-Hospital Reliability}

Of the 72 hospitals participating in Riksstroke, 67 recorded the 5 stroke test cases. Figure 3 shows the distribution of incorrect codings in the 81 variables in the Riksstroke acute care register. For a great majority of variables (67 of 81 ), the hospitals coded the variables correctly with only a few deviations $(0-5 \%$ incorrect codings). For 4 variables, there were more than $15 \%$ incorrect codings. These were delayed from onset to arrival in hospital, test of swallowing, day of stroke onset 


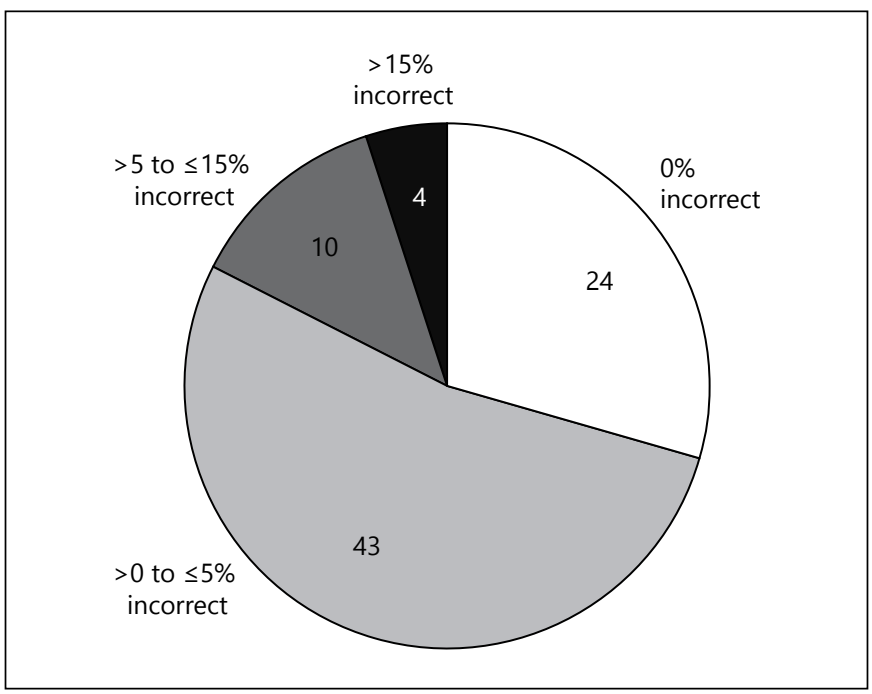

Fig. 3. Distribution of 81 variables in the Riksstroke acute care form by the proportion of incorrect codings in the validation of inter-hospital reliability (67 hospitals).

and CT angiography or MR angiography. The proportion of incorrect codings ranged from 0 to $7 \%$ between hospitals.

\section{Discussion}

Overall, these studies show that from different validation perspectives, a nationwide stroke care register like Riksstroke can be maintained with sufficient data quality to permit monitoring of the quality of care and benchmarking between hospitals. Thus, completeness and content validity are very satisfactory, and inter-hospital reliability is high.

To be able to make fair comparisons between hospitals, it is essential that the register covers all patients admitted for acute stroke. In the early years when Riksstroke was put to use, patients who died early soon after admission were often missed out in some hospitals $[16,17]$. This may have resulted in a spuriously low case fatality rate in these hospitals. In recent years, the coverage has been $90 \%$ when compared to the NPR. Since there is a considerable overdiagnosis of acute stroke in NPR (false positives), the actual completeness of Riksstroke is probably well above $90 \%$. A previous validation study showed false positive stroke diagnoses among patients recorded in Riksstroke to be $1.4 \%$ [16]. Thus, fair between-hospital benchmarking of the quality of acute stroke care seems possible. Although originally not intended for epidemio-

Validation of Hospital Performance

Measures in Stroke logical purposes, Riksstroke now also meets next-to-all criteria for an 'ideal' stroke epidemiological study, as defined by Sudlow and Warlow [18]. Riksstroke does not, however, cover out-of-hospital events. As summarized by Hallström et al. [19], a number of regional epidemiological studies have shown that home care for acute stroke is infrequent in Sweden; an exception may be institutionalised frail elderly people with multiple chronic disorders.

When considering what variables should be recorded in a quality assessment register, a balance must be struck between simplicity and content validity covering all important aspects of stroke care quality. Extensive recordings may deter less-enthusiastic hospitals, that is, hospitals mostly in need of monitoring the quality of care, from participating in the register. Our content validity studies show that Riksstroke includes next to all core variables that are identified by experts and other European registers, the exception in Riksstroke being acute administration of aspirin. The preconditions for collecting performance indictor data may vary greatly from country to country. The present Riksstroke form includes 81 items. This seems to be within the limits of what the stroke care staff can cope with (although there are frequent complaints about the administrative burden in Swedish health care). If more variables are considered to be added, their validity should be carefully tested in advance. Ongoing national work with automatic transfer of data from computerized medical records to national quality registers is intended to alleviate the burden of recording in Riksstroke and other national quality registers.

Coding of test cases is an inexpensive but underused method to estimate register data quality. The inter-hospital reliability was generally high for the items included in the Riksstroke acute care form. For a few items, however, the reliability was unsatisfactory, indicating that the instructions in the Riksstroke manual should be improved. The fact that none of the participating hospitals deviated much from the rest suggests that local educational activities on Riksstroke recording procedures are of satisfactory quality. The number of test cases was, however, low and it is possible that more test cases could have affected the figures on reliability (in either direction). The fact that this was a test exercise may also have affected the results.

Due to a very marked heterogeneity between European stroke registers in terms of what is recorded, the part of the content validity analyses that pertains to comparisons with other registers should be interpreted with caution.

The assessments of inter-hospital reliability were based on test cases with abbreviated medical records. Com- 
pared to regular cases, the coders therefore had more limited information and they may have had difficulties to find the necessary information. This may have resulted in a lower proportion of correct answers and, thus, an overestimate of the inter-hospital variability.

\section{Conclusions}

Our validation studies show that several aspects of the quality of a stroke care register can be assessed to be used for further development of the register. Although Riksstroke seems to perform satisfactorily as a register in most aspects, sometimes even excellent, our validations have identified some items that should be improved or deleted. Our experiences may serve as a model for other stroke registers in their efforts to evaluate their data quality. As data quality may change over time, it seems essential that repeated validations are incorporated in the management of stroke care performance registers.

\section{Acknowledgements}

Åsa Johansson, RN, and Sari Wallin, RN, contributed to the assessments of inter-hospital variability. Maria Sukhova, MSc, provided assistance in data management.

\section{References}

1 Katzan IL, Spertus J, Bettger JP, Bravata DM, Reeves MJ, Smith EE, et al: Risk adjustment of ischemic stroke outcomes for comparing hospital performance: a statement for healthcare professionals from the American heart association/American stroke association. Stroke 2014;45:918-944.

2 Fonarow GC, Reeves MJ, Smith EE, Saver JL, Zhao X, Olson DW, et al: Characteristics, performance measures, and in-hospital outcomes of the first one million stroke and transient ischemic attack admissions in Get-withthe-Guidelines - Stroke. Circ Cardiovasc Qual Outcomes 2010;3:291-302.

3 Meretoja A, Roine RO, Kaste M, Linna M, Juntunen M, Erilä T, et al: Stroke monitoring on a national level: PERFECT stroke, a comprehensive, registry-linkage stroke database in Finland. Stroke 2010;41:2239-2246.

4 Schwamm LH, Fonarow GC, Reeves MJ, Pan W, Frankel MR, Smith EE, et al: Get with the guidelines - stroke is associated with sustained improvement in care for patients hospitalized with acute stroke or transient ischemic attack. Circulation 2009;119:107-115.

5 Steiner MM, Brainin M; Austrian Stroke Registry for Acute Stroke Units: The quality of acute stroke units on a nation-wide level: the Austrian stroke registry for acute stroke units. Eur J Neurol 2003;10:353-360.

6 Asplund K, Hulter Åsberg K, Appelros P, Bjarne D, Eriksson $M$, Johansson $A$, et al: The Riks-stroke story: building a sustainable national register for quality assessment of stroke care. Int J Stroke 2011;6:99-108.
7 Cadilhac DA, Amatya B, Lalor E, Rudd A, Lindsay $\mathrm{P}$, Asplund $\mathrm{K}$ : Is there evidence that performance measurement in stroke has influenced health policy and changes to health systems? Stroke 2012;43:3413-3420.

8 Wiedmann S, Hillmann S, Abilleira S, Dennis M, Hermanek P, Niewada M, et al: Variations in acute hospital stroke care and factors influencing adherence to quality indicators in 6 European audits. Stroke 2015;46:579-581.

9 Hokstad A, Indredavik B, Bernhardt J, IhleHansen $H$, Salvesen $\varnothing$, Seljeseth YM, et al: Hospital differences in motor activity early after stroke: a comparison of 11 Norwegian stroke units. J Stroke Cerebrovasc Dis 2015; 24:1333-1340.

10 Langley GJ, Moen R, Nolan KM, Nolan TW, Norman CL, Provost LP: The Improvement Guide: A Practical Approach to Enhancing Organizational Performance, ed 2. Hoboken, Jossey-Bass, 2009.

11 Norrving B, Wester P, Sunnerhagen KS, Terént A, Sohlberg A, Berggren F, et al: Beyond conventional stroke guidelines: setting priorities. Stroke 2007;38:2185-2190.

12 National Board of Health and Welfare. The National Patient Register, https://www. socialstyrelsen.se/register/halsodata register/patientregistret/inenglish (accessed on August 28, 2015).
13 Köster M, Asplund K, Johansson Å, Stegmayr B: Refinement of Swedish administrative registers to monitor stroke events on the national level. Neuroepidemiology 2013;40:240246.

14 Norrving B, Bray BD, Asplund K, Heuschmann P, Langhorne P, Rudd AG, et al: Cross-national key performance measures of the quality of acute stroke care in Western Europe. Stroke 2015;46:2891-2895.

15 Wiedmann S, Norrving B, Nowe T, Abilleira S, Asplund K, Dennis M, et al: Variations in quality indicators of acute stroke care in 6 European countries: the European implementation score (EIS) collaboration. Stroke 2012;43:458-463.

16 Glader EL: Stroke Care in Sweden. Hospital Care and Patient Follow-Up Based on RiksStroke, the National Quality Register for Stroke Care. Umeå University Medical Dissertations, 2003.

17 Appelros $\mathrm{P}$, Högerås N, Terént A: Case ascertainment in stroke studies: the risk of selection bias. Acta Neurol Scand 2003;107:145149.

18 Sudlow CL, Warlow CP: Comparing stroke incidence worldwide: what makes studies comparable? Stroke 1996;27:550-558.

19 Hallström B, Jönsson AC, Nerbrand C, Petersen B, Norrving B, Lindgren A: Lund stroke register: hospitalization pattern and yield of different screening methods for firstever stroke. Acta Neurol Scand 2007;115:4954. 